\title{
Stress-induced takotsubo cardiomyopathy in survivors of the 2011 Queensland floods
}

Stuart J Butterly BSc(Hons), MB BS, FRACP, Fellow in Interventional Cardiology, and PhD Candidate

Mathivathana Indrajith

BSc(Hons), MB BS, MRCP Advanced Trainee,' and MPhil Candidate

Paul Garrahy MB BS, FRACP Cardiologist

Arnold C T Ng MB BS, PhD, FRACP. Cardiologist,' and Senior Lecturer $^{2}$

Paul A Gould MB BS, PhD, FRACP Cardiologist, and Associate Professor

William Y S Wang MM BS, PhD, FRACP Cardiologist, and Associate Professor

1 Department of Cardiology, Princess Alexandra Hospital, Brisbane, QLD.

2 School of Medicine niversity of Queensland Brisbane, QLD.

stuart.butterly@ uq.net.au

MJA 2013; 198: 109-110 doi: 10.5694/mjal2.11620

\section{We report the first two Australian cases of flood-related takotsubo cardiomyopathy, in patients who were trapped by life-threatening flash floods in and near Toowoomba, Queensland. It is well recognised that events that incite significant physical and emotional stress in individuals can trigger this syndrome, which often mimics acute myocardial infarction.}

\section{Clinical records}

\section{Patient 1}

On 10 January 2011, at about 13:30, the Queensland city of Toowoomba experienced flash flooding caused by intense rainfall over the catchment areas of three creeks that traverse the city. ${ }^{1}$ When the central business district was hit by "a wave of water", a 66-year-old female resident became trapped in her flooded and spinning car. She was removed from her car by witnesses. That evening she developed chest pain with associated shortness of breath and palpitations. The pain came and went throughout the night before she attended the local hospital.

Her background medical history included asthma, gastro-oesophageal reflux disease, L4-L5 intervertebral disc prolapse and generalised anxiety disorder. She had ceased smoking 6 years previously after 42 pack-years.

On arrival at the hospital she was afebrile, had a regular heart rate (70 beats per minute), a respiratory rate of 20 breaths per minute and blood pressure of $135 / 80 \mathrm{mmHg}$. Pulse oximetry indicated her arterial oxygen saturation was $94 \%$ at rest. She was overweight. Her jugular venous pressure was $4 \mathrm{~cm}$ above the sternal angle. There were two normal heart sounds without extra sounds. Expiratory wheeze and decreased air entry were noted over hyperexpanded lung fields. There was evidence of a minor increase in accessory respiratory muscle use. Examination of other systems was unremarkable. An electrocardiogram (ECG) showed sinus rhythm and normal cardiac axis, PR interval and QRS duration. There was T-wave inversion in leads II, III, aVF and V3-V6. The patient's serum troponin I level was

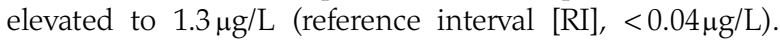
Results of biochemistry and haematology testing were otherwise unremarkable.

Her respiratory signs improved quickly with administration of inhaled bronchodilators, and she was transferred to our tertiary hospital.

Transthoracic echocardiography demonstrated severe left ventricular systolic dysfunction with akinesis and ballooning of the apical segments and preserved contractility of the basal segments. Invasive coronary angiography revealed no evidence of coronary artery obstruction. Left ventriculography confirmed ballooning of the apex.
Given the acute stressor event, the elevated cardiac enzyme level, the absence of significant coronary artery stenosis and an apical ballooning pattern of left ventricular dysfunction, we established a diagnosis of takotsubo cardiomyopathy. The patient improved clinically with supportive treatment during her inpatient stay and was discharged on Day 4 with evidence of early recovery of left ventricular systolic function. It was not possible to arrange further follow-up investigations.

\section{Patient 2}

Patient 2 was a 68-year-old female resident of the Lockyer Valley, located about 10 kilometres to the east of Toowoomba. At about 14:00 on 10 January 2011, the region experienced extremely rapid flooding of sufficient strength to carry cars. ${ }^{1}$

The patient and her husband were trapped in their house when flood waters hit, eventually escaping by breaking a window. They held onto a large bird cage until the water receded to avoid being swept away. At about 02:30 the next morning, the patient began to experience chest pain that she described as a "rock in the chest", associated with shortness of breath. She subsequently presented to the local hospital. The pain did not abate with supplemental oxygen and sublingual glyceryl trinitrate, but resolved spontaneously at 09:00.

The patient was overweight and had a background of treated glaucoma, gastro-oesophageal reflux disease and osteoarthritis. She had no history of coronary artery disease or chest pain syndrome.

On presentation, she was afebrile with a regular heart rate (82 beats per minute), blood pressure of $105 / 65 \mathrm{mmHg}$ and arterial oxygen saturation measured by pulse oximetry of $99 \%$ on room air. Her jugular venous pressure was $3 \mathrm{~cm}$ above the sternal angle, and normal first and second heart sounds were present without murmurs or added heart sounds. Adequately ventilated lung fields free of crepitations, rales or crackles were noted. Examination of other systems was unremarkable. An ECG confirmed sinus rhythm (86 beats per minute) and normal cardiac axis and P-wave and QRS-complex morphology. PR and QRS intervals were within normal limits. The corrected QT interval was prolonged $(507 \mathrm{~ms}[\mathrm{RI},<450 \mathrm{~ms}])$. Chest x-ray 
showed minor prominence of the pulmonary vasculature without significant signs of heart failure.

Peripheral blood assessment was significant for an elevated white cell count of $15.1 \times 10^{9} / \mathrm{L}$ (RI, $4.0-11.0 \times 10^{9} / \mathrm{L}$ ) with neutrophilia (neutrophil count, $11.61 \times 10^{9} / \mathrm{L}$ [RI, $\left.2.00-8.00 \times 10^{9} / \mathrm{L}\right]$ ) and a mildly depressed estimated glomerular filtration rate (eGFR) of $56 \mathrm{~mL} / \mathrm{min} / 1.73 \mathrm{~m}^{2}$ (RI, $>60 \mathrm{~mL} / \mathrm{min} / 1.73 \mathrm{~m}^{2}$ ). The patient's troponin I level was elevated $(5.0 \mu \mathrm{g} / \mathrm{L})$, as was her B-type natriuretic peptide level (1320 ng/L [RI, <100 ng/L]).

The patient was transferred to our tertiary hospital for ongoing assessment and management.

Selective coronary angiography revealed no significant coronary artery stenosis. Left ventriculography demonstrated left ventricular systolic dysfunction with severe hypokinesis and apical ballooning (Box, A). Transthoracic echocardiography confirmed the left ventriculography findings. There was no evidence of myocardial infarction on cardiac magnetic resonance imaging. We made a diagnosis of takotsubo cardiomyopathy.

The patient was stable during her inpatient stay. Followup clinical and echocardiography assessment established interval improvement in left ventricular systolic function, and her recovery was complete 2 months after presentation. She has remained well at 1 year.

\section{Discussion}

We report the first two Australian cases of flood-related takotsubo (stress-induced) cardiomyopathy, which occurred during the 2011 Queensland floods.

Takotsubo cardiomyopathy is an increasingly recognised phenomenon that often mimics acute myocardial infarction. It is characterised by a combination of chest pain, progressive ECG abnormalities (most commonly T-wave inversion and QT-interval prolongation), elevated levels of cardiacspecific biomarkers (troponin I and creatine kinase), angiographically normal coronary arteries and apical ballooning seen on echocardiography and left ventriculography.

The syndrome was first reported in the Japanese cardiology literature in $1991 .^{2}$ It is named for the resemblance of left ventricle morphology on ventriculography during the acute phase to that of a Japanese octopus trap and is synonymous with the terms apical ballooning syndrome or ampulla cardiomyopathy. Since the initial description, at least two other accepted ballooning patterns (mid ventricular and basal) have been described. ${ }^{3}$

Takotsubo cardiomyopathy is colloquially known as "broken heart syndrome" or "stress cardiomyopathy", acknowledging its frequent association with extreme emotional or physical stressors. The exact mechanism of the acute cardiomyopathy is unclear, but is likely to be secondary to abnormal responses to acute catecholamine surges. Examples of physical stressors include acute respiratory illnesses, administration of exogenous catecholamines (adrenaline, noradrenaline, or dobutamine in patients undergoing stress echocardiography), and acute subarachnoid haemorrhage. Takotsubo cardiomyopathy may occur in patients under general anaesthesia, suggesting an autonomic nervous system mechanism. ${ }^{4}$ The syndrome most often affects postmenopausal women; ;,4 up to $90 \%$ have an identifiable stressful trigger event. ${ }^{4}$ Complete
End systolic left ventriculogram of Patient 2 during her acute presentation (A), showing apical ballooning; and a normal left ventriculogram (B)



recovery from the syndrome is observed in nearly all cases (96\%) within 7 to 37 days. ${ }^{5}$ Recurrence rates are reported to be between $5 \%$ and $10 \% .{ }^{6}$ Mortality rates are low and published cases of death are rare.

Reported rates of acute coronary syndromes show increases after natural disasters. Onset of myocardial infarction is associated with acute physiological stress, from everyday triggers including the circadian rhythm (peak prevalence in the morning hours) to physical activity and severe meteorological events. ${ }^{7}$ Stressor events increase sympathetic activation of the autonomic nervous system, increasing blood pressure, heart rate, myocardial contractility and, consequently, myocardial oxygen demand. They are also associated with increased platelet aggregability, other prothrombotic changes, and altered intravascular sheer stress, which may disrupt atherosclerotic plaques, resulting in coronary artery occlusion and ischaemia.

Until very recently, the link between takotsubo cardiomyopathy and natural disasters had received little attention, ${ }^{8}$ although increased prevalence of acute coronary syndrome presentations coinciding with extreme natural events has been previously documented. ${ }^{9}$ Given that takotsubo cardiomyopathy has been under-recognised until recently and that it mimics myocardial infarction and coronary artery spasm, we suspect that takotsubo cardiomyopathy may explain some of the historically recognised instances of increased acute coronary syndromes during and shortly after natural disasters.

Competing interests: No relevant disclosures.

Received 5 Nov 2012, accepted 8 Jan 2013.

1 Holmes CE. Queensland Floods Commission of Inquiry. Final report. Brisbane: Queensland Government, 2012. 2 Dote K, Sato H, Tateishi H, et al. [Myocardial stunning due to simultaneous multivessel coronary spasms: a review of 5 cases] [Japanese]. J Cardiol 1991; 21: 203-214.

3 Eitel I, von Knobelsdorff-BrenkenhoffF, Bernhardt P, et al. Clinical characteristics and cardiovascular magnetic resonance findings in stress (takotsubo) cardiomyopathy. JAMA 2011; 306: 277-286.

4 Sharkey SW, Windenburg DC, Lesser JR, et al. Natural history and expansive clinical profile of stress (takotsubo) cardiomyopathy. J Am Coll Cardiol 2010; 55: 333-341.

5 Pernicova I, Garg S, Bourantas CV, et al. Takotsubo cardiomyopathy: a review of the literature. Angiology 2010; 61: 166-173.

6 Bybee KA, Kara T, Prasad A, et al. Systematic review: transient left ventricular apical ballooning: a syndrome that mimics ST-segment elevation myocardial infarction. Ann Intern Med 2004; 141: 858-865.

7 Culic V, Eterovic D, Miric D. Meta-analysis of possible external triggers of acute myocardial infarction. Int J Cardiol 2005; 99: 1-8.

8 Trebouet E, Prieur S, Dimet J, et al. Cardiovascular emergencies related to the Xynthia storm. Am J Emerg Med 2012; 30: 377-379.

9 Watanabe H, Kodama M, Okura Y, et al. Impact of earthquakes on Takotsubo cardiomyopathy. JAMA 2005; 294: 305-307. 